\title{
Numerical simulation of extruded clay paste compression
}

\author{
Jérémie Vignes • Fabrice Schmidt • Gilles Dusserre • \\ Jean Frédéric Dalmasso
}

\begin{abstract}
The manufacturing process of clay tiles includes a pressing step in which the material undergoes stresses, that may result in the appearance of defects. To understand the phenomena involved, a numerical model of the pressing step was developed. Different tests were performed to determine the different behaviour laws necessary to the numerical simulation (rheological, tribological, damage). A rheological study, based on free compression tests, allowed to charaterize the elasto-visco-plastic behaviour of the extruded clay paste. The constitutive parameters were estimated by inverse analysis of the experimental force displacement curves using a Strategic evolution algorithm coupled with a metamodel. Two damage models, the Latham and Crockoft criterion and the Oyane criterion, were compared to model the cracking. To simulate the crack's propagation, an element deletion algorithm was used. The friction models of Coulomb and Tresca were investigated to model the global friction between the clay and the tools. The different parameters of the friction law were identified by inverse analysis of an experimental pressing force obtained during a shaping test. The identified model is valided on the case study of an instrumented forming of a tile lug and allows to simulate the shaping of an industrial tile.
\end{abstract}

J. Vignes $(\triangle) \cdot$ F. Schmidt $\cdot$ G. Dusserre

Université de Toulouse; Mines Albi, INSA, UPS, ISAE,

ICA (Institut Clément ADER), Campus Jarlard,

81013 Albi cedex 09, France

e-mail: jeremie.vignes@mines-albi.fr

F. Schmidt

e-mail: fabrice.schmidt@mines-albi.fr

G. Dusserre

e-mail: gilles.dusserre@mines-albi.fr

J. F. Dalmasso

CRED Terreal, Route de Revel, 11400 Castelnaudary, France

e-mail: jean-frederic.dalmasso@terreal.com
Keywords Clay forming - Numerical simulation .

Constitutive law · Free compression test · Inverse analysis

$\begin{array}{ll}\text { Nomenclature } \\ \mathrm{E} & \text { Young's modulus } \\ \mathrm{K}_{0} & \text { Consistency } \\ \mathrm{m} & \text { Strain rate sensitivity parameter } \\ \overline{\mathrm{m}} & \text { Tresca's friction parameter } \\ \mu & \text { Coulomb's friction parameter } \\ \mathrm{n} & \text { Strain hardening sensitivity parameter } \\ \mathrm{p} & \text { Hydrostatic pressure } \\ \bar{\varepsilon}_{\mathrm{R}} & \text { Equivalent plastic strain } \\ \bar{\varepsilon}_{\mathrm{V}} & \text { Equivalent viscoplastic strain } \\ \overline{\bar{\varepsilon}}_{\mathrm{V}} & \text { Equivalent viscoplastic strain rate } \\ \overline{\bar{\varepsilon}}_{\mathrm{E}} & \text { Elastic strain tensor } \\ \overline{\overline{\mathrm{I}}}_{\mathrm{V}} & \text { Identity tensor } \\ \nu & \text { Poisson's ratio } \\ \sigma_{\mathrm{I}}, \sigma_{\mathrm{II}}, \sigma_{\mathrm{III}} & \text { Principal stresses } \\ \sigma_{0} & \text { Flow stress } \\ \sigma_{\mathrm{n}} & \text { Normal stress } \\ \bar{\sigma} & \text { Von Mises stress } \\ \overline{\bar{\sigma}} & \text { Stress tensor } \\ \tau & \text { Shear stress } \\ t r() & \text { Mathematical function Trace }\end{array}$

\section{Introduction}

Clay pressing is the main process used to form clay tiles. The manufacturing process of clay tiles can be divided into five steps: raw material preparation, extrusion, pressing, and finally drying and firing. During the forming of a clay tile by pressing, the clay obviously undergoes stresses whose consequences can be cracks, pulling out, and warpage occurence. It is a major challenge to limit the appearance of defects, in 
particular during the forming step, in order to improve the process efficiency.

In this study, we have developed both a method to estimate the tribo-rheological parameters and a simplified numerical model of clay paste damaging during the tile pressing. Different experimental setups have been designed to identify the behaviour of the clay. As explained by Foudrinier [9], it is essential to perform tests involving a representative state of stress in order to determinate the behaviour of the clay. The results of Baran et al. [3] showed that the compression test allows to estimate the rheological parameters of a clay paste and in particular the plasticity limit. The rheological tests carried out by Ribeiro et al.[16]) revealed an elasto-plastic behaviour of different types of clay. However, after their studies of different types of Togo clay, Gagou et al. [10] highlighted that the choice of a viscoplastic model may be limited. Thus, it has been decided to model the clay paste behaviour using an elasto-visco-plastic law.

Compression tests are not well suited for the identification of the friction law between the clay and the tools surfaces. Indeed, it is required to measure the contour variations of the sample during the test. In material forming, two types of test are commonly used to characterize the interface between the formed material and the tools. The first one, presented in the paper, consists to measure directly the friction parameter on a simplified forming test which reproduces a state of stress and strain similar to those involved in the actual process. The identified parameters are some mean values of all the contact conditions. This method is also used to identify the friction behaviour in extrusion process [4]. The second one consists to characterize the friction under controlled contact conditions using a tribometer. This behaviour sensitive method provides more accurate results since the effect of the rheological behaviour are minimized. Djelal [6]) designed a tribometer for the study of the friction between clay mixture and a metallic surface. This experimental setup was used by Kocserha and Gömze [12] to study the effect of the clay compound on the friction behaviour. To model the tribological behaviour, different models exist. The main ones are the Coulomb model and the Tresca model. Some other models exist and offer some continous transition between this two models like the Orowan's friction model presented by Aydin et al. [2].

To model the damage, several laws are available. Cockroft and Latham [5]) have proposed a criterion that allows to take into account the tensile stress limit. In their research works, Ribeiro et al. [16] explained that the occurence of cracks during a compression test was due to the failure of the aqueous bridge between two particules (liquid film surrounding the clay particles). The capillary forces involved allow to keep the particles together during the deformations. When the tensile stress exceeds the magnitude of the capillary force, the link between the particles are removed leading a crack to initiate. Soulie [17] proposed a model which allows to take into account the capillary force according to the particles geometry and the interstitial distance. Lian et al. [14] defined a damage criterion based on a maximal distance between two particles. These interpretations of the phenomena at microscale level are consistent with a macroscopique criterion based on the tensile strain.

The different parameters of the rheological and tribological laws were identified using an inverse analysis approach. The compression test can be easily schematized in a $2 \mathrm{D}$ axisymetric configuration. The analytical solutions given by Engmann et al.[8]) allow to calculate the squeezing force according to the different parameters of the behaviour laws. The Strategic Evolution algorithm, developed by Emmerich et al. [7], available in the optimization module of the commercial software Forge $2009^{\circledR}$ allows to identify the parameters of the constitutive law.

The numerical simulation was performed using the identified behaviour and damage models. The cracks were simulated using an element deletion algorithm presented by Lee et al. [13].

The goal of this study is to simulate the shaping step of a tile in "closed tools" using Finite Element method. The beginning of this paper is devoted to the presentation of the material and the different tests performed. The triborheological and damage behaviour identified using these tests are then presented. Finally, an experimental shaping test is compared to its numerical simulation. For sake of confidentiality, we are not allowed to show the industrial geometry of a tile. The geometry presented in the paper, is a case study developed to obtain a state of stress similar to those involved during the industrial pressing step.

\section{Material and tests}

The material under study is a clay paste. Its composition, detailed in Table 1, is a mixture of clay and sand (temper), with a water content around $20 \%$ of the dry mass. The sand function is to limit the shrinkage, and thus the cracking during the drying and the firing steps of the manufacturing process.
Table 1 Composition of the clay paste

\begin{tabular}{lllll}
\hline Material & Clay (\%) (Illite and kaolinite) & Sand (\%) & Water ( \% of dry weight) & Additives \\
\hline A & 69 & 31 & 20,5 & No \\
\hline
\end{tabular}




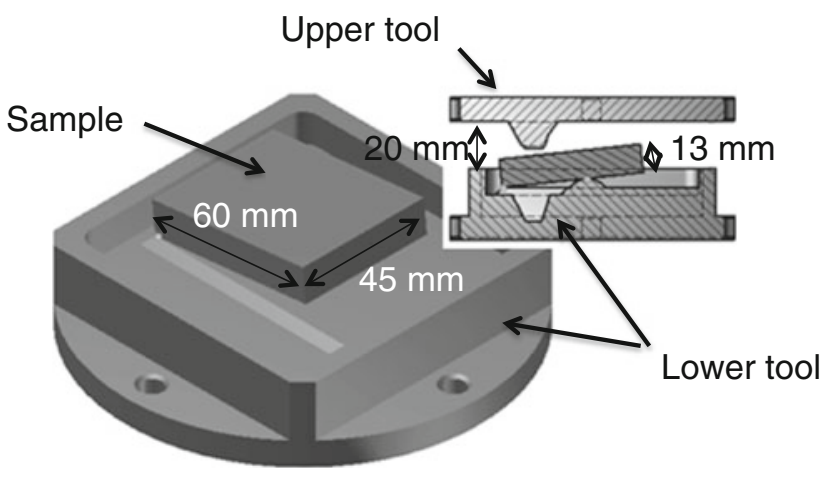

Fig. 1 View of the tools and samples of the technological test

During the firing step, the sand grains form the nodes of a network which gives the final mechanical properties to the tile. The amount of this component must be carefully chosen: too much temper decreases the clay plasticity and formability.

In the industrial process, the raw plate to be pressed is obtained by extrusion of the mixture exposed to vacuum to suppress the porosities in the material. In this work, a homogeneous material without porosity is assumed because the material is extruded with a low air pressure.

The rheological behaviour was investigated by compression tests. Using this set-up, the resulting distribution of stresses is very similar to those involved in the pressing step.

The tests were performed thanks to an electro-mechanical testing machine, with a $30 \mathrm{kN}$ load cell. A wide range of strain rates were investigated by setting three constant crosshead displacement rates: $0,1 \mathrm{~mm} \cdot \mathrm{s}^{-1} ; 1 \mathrm{~mm} \cdot \mathrm{s}^{-1} ; 8.5 \mathrm{~mm} \cdot \mathrm{s}^{-1}$. The cylindrical samples were $60 \mathrm{~mm}$ diameter and $22 \mathrm{~mm}$ thick. The processing of compression tests data is direct only if the contact between the sample and the plates is known. The determination of the different parameters with a free compression test is difficult when the friction between the tools and the sample is not know. In order to avoid this problem, the pressing plates were modified in order to ensure a quasisticky contact. The tools were thus covered with sand paper

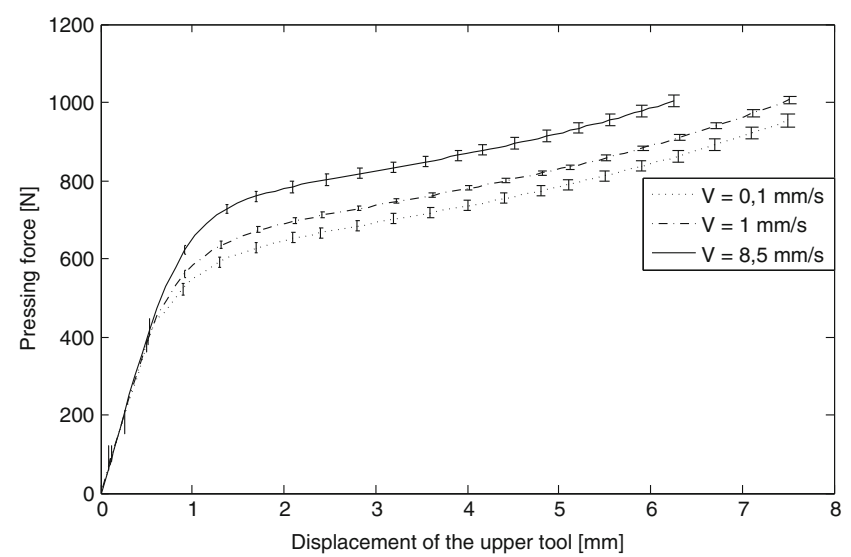

Fig. 2 Compression force during compression tests at different crosshead displacement rates
Table 2 Parameters of the constitutive law

\begin{tabular}{ll}
\hline Material & A \\
\hline Young's Modulus (MPa) & 3,84 \\
Consistency (MPa.s) & 0,194 \\
Strain rate sensitivity & 0,03 \\
\hline
\end{tabular}

to create a sticky contact between the tools and the clay paste (average particles diameter of $190 \mu \mathrm{m}$ ).

The variation of the sample boundary could be analysed to determinate the friction parameter but this method is difficult to set up and is not enough sensitive to friction to characterize the friction behaviour. So, we have decided to perform a new compression test on technological tools allowing to stamp a tile lug. The tools and the clay sample are presented in the Fig. 1.

For this test, we pressed a small clay sheet in a prototype reproducing the strains path involved in the forming of an industrial tile. The geometry of the tools allows to stamp a parallelepiped sample. The tools were made with stainless
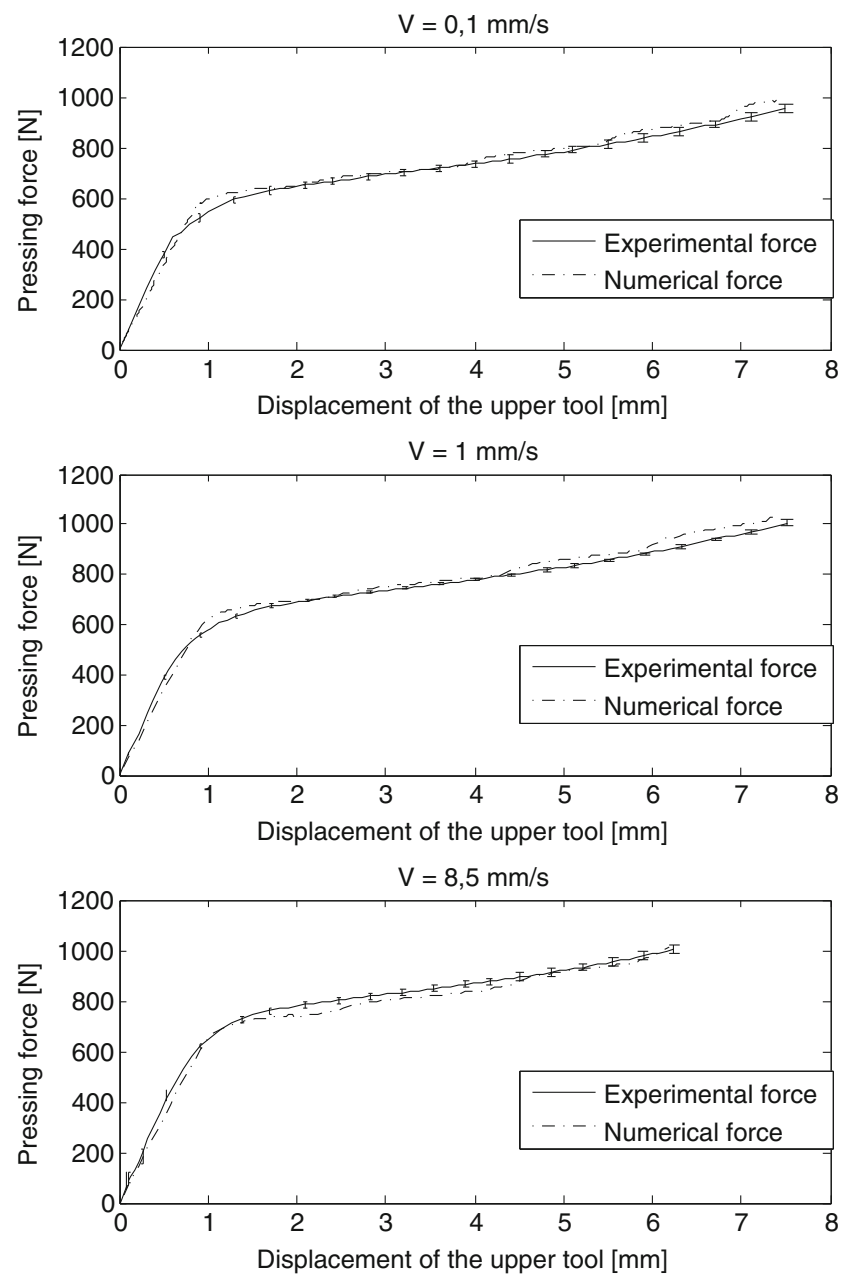

Fig. 3 Comparison of the computed and experimental squeezing forces for different crosshead displacement rates 


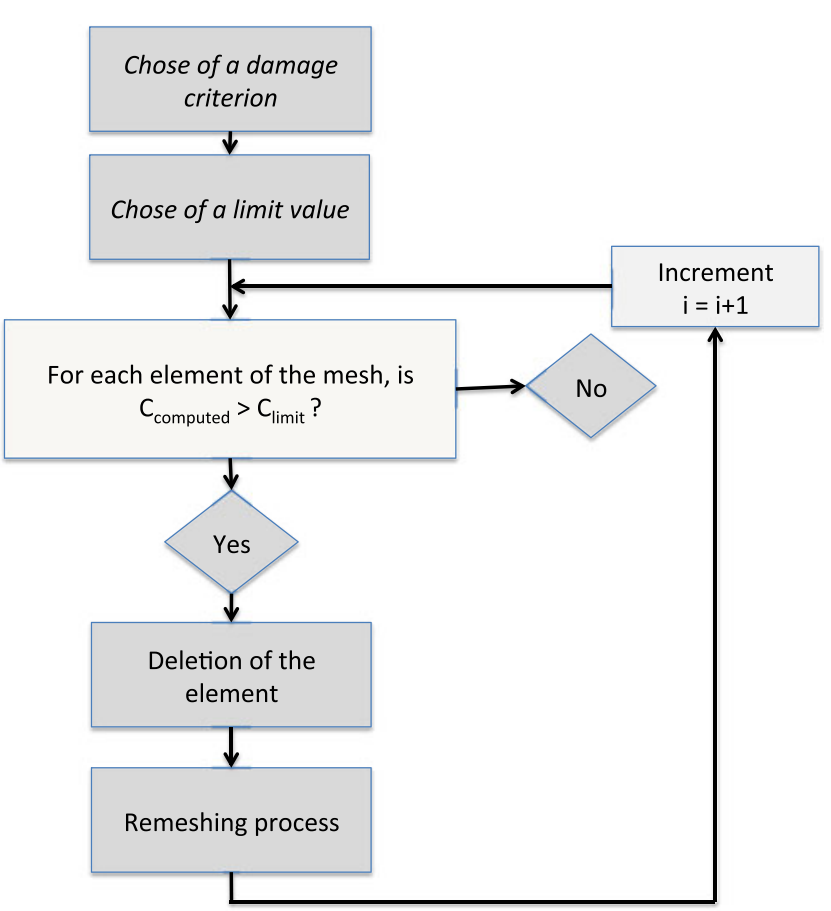

Fig. 4 Element deletion algorithm

steel. The areas in contact with the clay were polished to obtain the roughness of surface like industrial tools. The shaping tests were performed with a upper tool displacement rate of $5 \mathrm{~mm} / \mathrm{s}$.

\section{Experimental results and identification of the rheological and damage behaviour}

Rheological behaviour

The free compression test confirmed that the clay paste behaves as an elasto-visco-plastic behaviour.

Figure 2 represents the variation of the squeezing force versus the crosshead displacement for three compression velocities.

The average experimental curves are plotted with the minimal and the maximal values measured during five tests in the

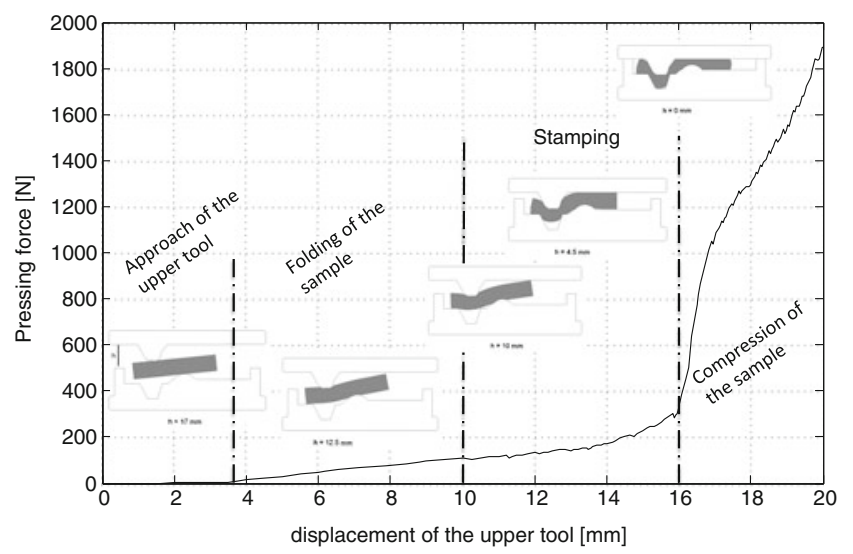

Fig. 6 Variation of the pressing force according to the displacement

same conditions showing that the repeatability of the test is fair. The curves can be divided into an elastic part and a viscoplastic part. The elastic range follows the generalized Hooke's law :

$\overline{\bar{\varepsilon}}_{\mathrm{E}}=\frac{1+v}{\mathrm{E}} \overline{\bar{\sigma}}-\frac{v}{\mathrm{E}} \operatorname{tr}(\overline{\bar{\sigma}}) \overline{\overline{\mathrm{I}}}$

The evolution law of the flow stress is defined by Eq. 2 .

$\bar{\sigma}=\sigma_{0}+\sqrt{3 \mathrm{~K}_{0}}\left(\bar{\varepsilon}_{\mathrm{V}}\right)^{\mathrm{n}}\left(\sqrt{3 \dot{\bar{\varepsilon}}_{\mathrm{V}}}\right)^{\mathrm{m}}$

where the Von Mises yield criteria was used.

In order to determine the different parameters of the constitutive law, we used a Strategic Evolution Algorithm [11]. This global optimisation algorithm is based on the evolution of a population of individuals. The algorithm can be decomposed into five steps. The first step is the initialisation step with the creation of an initial population. In the next step, the quality of each individual of the population is evaluated. The individuals that minimise the cost function is conserved and incurred, in the thirdstep, to create a population of descendants. Next, the descendants are evaluated. The fifth step is a comparison step. If a descendant is better, he substitutes for its parent. A new population is obtained. The algorithm starts again at the second step until the maximum iteration
Fig. 5 Evolution of the mesh during the compression test

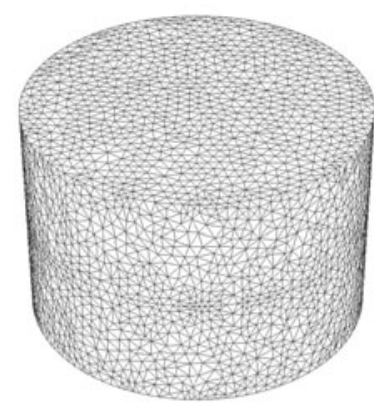

Initial mesh : 53700 elements

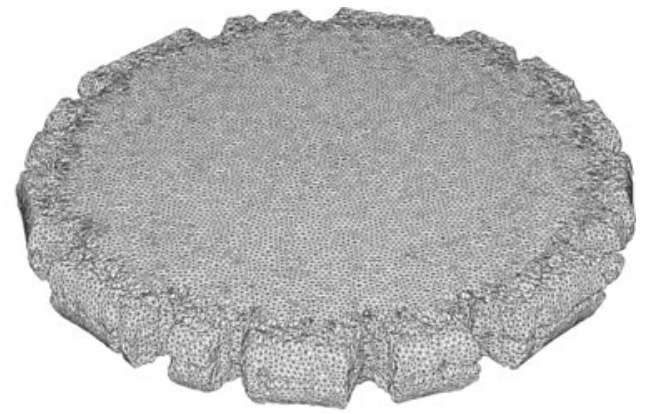

Final mesh : 650000 elements 
Table 3 Friction parameters

\begin{tabular}{llll}
\hline Friction law & Coulomb & Tresca & Coulomb-Tresca \\
\hline$\mu$ & 0,318 & $\mathrm{X}$ & 0,47 \\
$m$ & $\mathrm{X}$ & 0,76 & 1,73 \\
\hline
\end{tabular}

number is reached. This algorithm allows to arise a global minimum but the accuracy is lower than a local algorithm.

The parameters were identified from the experimental force/displacement curves for three crosshead displacement rates. The identification range was limited to the first 15 millimeters of squeezing. This allows to obtain the constitutive law before the cracking.

The rheological parameters identified were the Young's modulus $E$, the consistency $K_{0}$, the strain rate sensitivity $m$ and the strain hardening sensitivity $n$. The Poisson's ratio was fixed at 0,45 , as it is commonly given in the literature [1]. A set of parameters was identified for each crosshead displacement rate. The mean value of each evaluation was finally calculated for each parameter in order to take into account the whole experienced strain rate range. The results are reported in Table 2.

The strain rate parameter is low but it is important to take into account the effect of the velocity. The compression tests also reveal a low dependance of the rheogical law with the strain hardening. However, this parameter allows a better agreement between the numerical and experimental strength / displacement curves.

Figure 3 compares the experimental force / displacement curves to the simulated one obtained with the identified set of parameters.

The numerical data were computed using the commercial software Forge 2009. The accuracy of the model given by the optimisation algorithm can be controlled by calculations of the mean squared error between the two curves. For the model presented, the mean squared error was about $3 \%$.

\section{Damage behaviour}

In order to model the damaging of the clay, an elements deletion algorithm [13] was used in the numerical model. The Fig. 4 presents the elements deletion algorithm.

The first step consists to define a damage criterion and the failure threshold.

At each step of computation, the value of the criterion is evaluated on each element of the mesh. If the value computed is higher than the failure threshold, the element is deleted. This method allows to model easily the cracks initiation and propagation. However, the mesh around the cracks needs to be refined to limit the loss of volume resulting from the elements deletion and affects the crack propagation.

To model the damage, two criteria were tested. The first one was the Latham and Cockroft criterion defined by Eq. 3:

$\mathrm{C}_{L C}=\int_{0}^{\bar{\varepsilon}_{\mathrm{R}}} \frac{\operatorname{Max}\left(\sigma_{\mathrm{I}}, \sigma_{\mathrm{II}}, \sigma_{\mathrm{III}}\right)}{\bar{\sigma}} \mathrm{d} \bar{\varepsilon}$

The second one was the Oyane criterion [15] defined by Eq. 4:

$\mathrm{C}_{O}=\int_{0}^{\bar{\varepsilon}_{\mathrm{R}}}\left(1+C_{1^{-}}^{\underline{p}}\right) \mathrm{d} \bar{\varepsilon}$ if $p<0$ (tensile stress)

For each test, the sample height $H_{d}$ where the material begins to damage has been measured. The failure threshold is defined as the value of the criterion reached in the simulation when the height of the sample is $H_{d}$. The value of the failure threshold is 0.35 in the case of the Latham and Cockroft criterion. For the Oyane criterion, we set $C_{1}=0$ to obtain a criterion based only on a critical strain. We obtained a failure threshold value around 0.25 . To limit the number of elements and control the CPU times, we decided to refine the
Fig. 7 Numerical and experimental pressing force for three different friction conditions

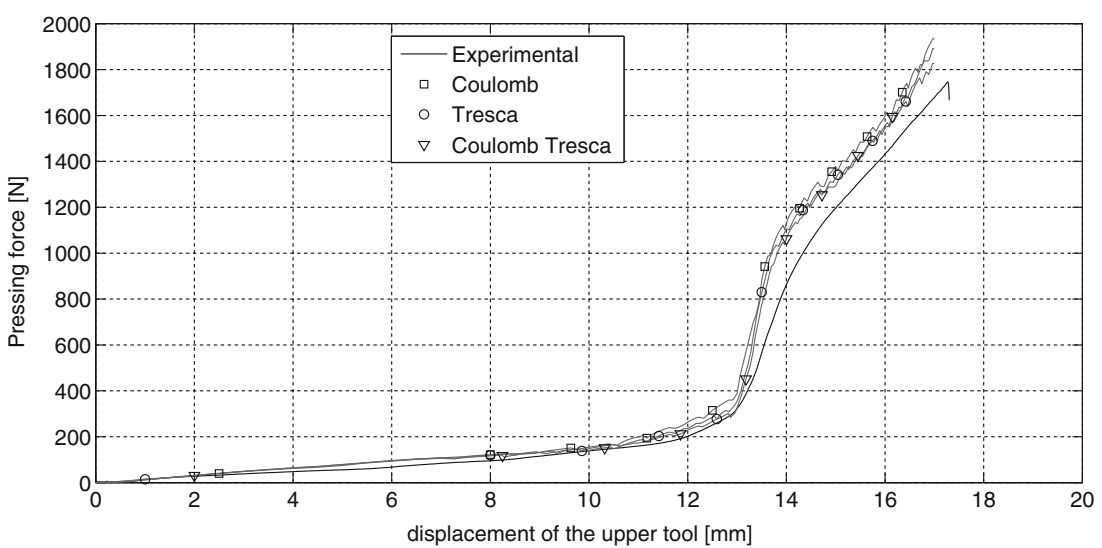


Fig. 8 Comparision between two technics of meshing

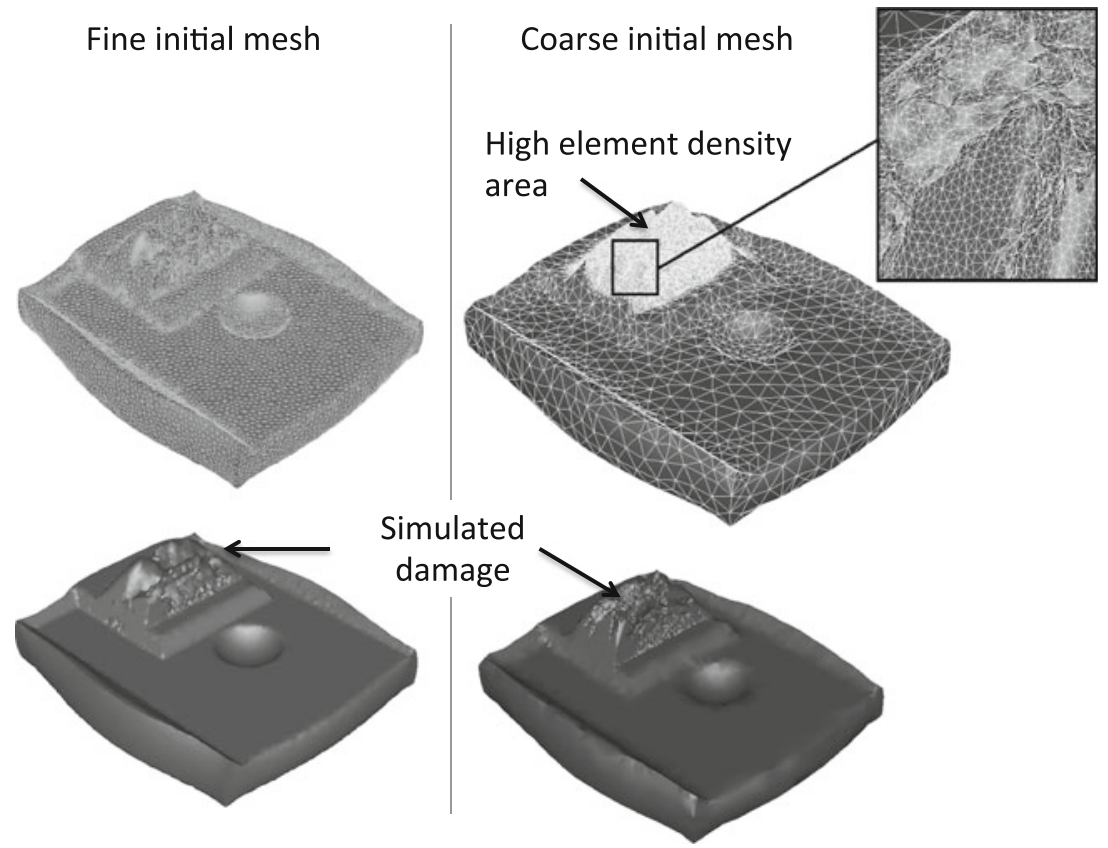

mesh only when the value of the damage criterion becomes higher than $17 \%$ of the failure threshold.

Figure 5 shows the mesh of a numerical sample at the beginning and at the end of the compression test.

The mesh was refined during the simulation. The two criteria can reproduce the initiation and the propagation of the cracks around the sample. Whereas damaging starts on weaknesses related to the material heterogeneities, the model describes a heterogeneous criterion field due to the finite element discretization. The result obtained was very sensitive to the mesh size, and a very fine mesh is required to provide a crack pattern similar to the experimental one. Moreover, the element deletion method is not well suited to model the crack propagation because of a bad evaluation of the stress intensity at the crack tip. It results in a fracture pattern not so sharp as observed experimentally.

\section{Shaping with technological tools}

Friction law identification

As it was explained previously, the identification of a friction law with a compression test is tedious. So it was decided to perform a compression test on technological tools. The objective is to determine an overall friction law between the clay and the tools during the shaping step. Three friction models can be considered.

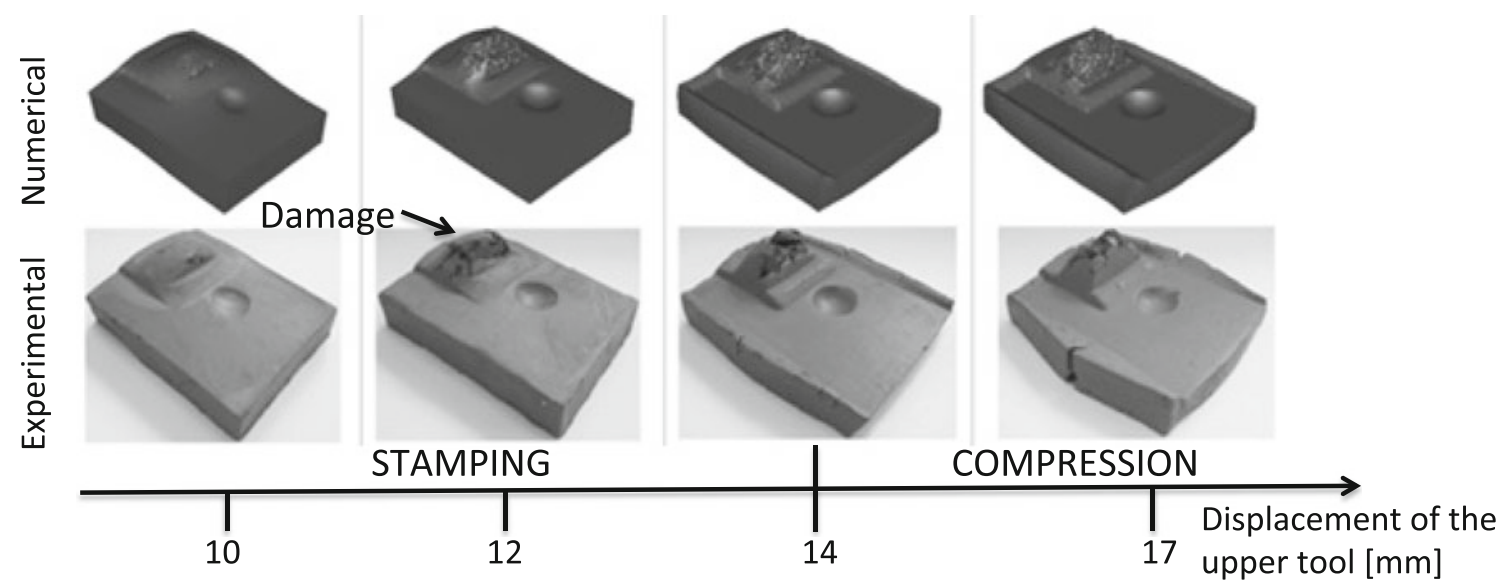

Fig. 9 Evolution of the cracks on the lug 
The first model assumes the Coulomb law given by the Eq. 5:

$$
\tau=\mu \sigma_{n}
$$

The second model assumes a Tresca law defined by the following equation:

$\tau=\bar{m} \frac{\sigma_{0}}{\sqrt{3}}$

The last model was a mix of the two others. It follows the Coulomb limited Tresca law (Eq. 7):

$\left\{\begin{array}{l}\tau=\mu \sigma_{n} \text { if } \mu \sigma_{n}<\bar{m} \frac{\sigma_{0}}{\sqrt{3}} \\ \tau=\bar{m} \frac{\sigma_{0}}{\sqrt{3}} \text { if } \mu \sigma_{n} \geq \bar{m} \frac{\sigma_{0}}{\sqrt{3}}\end{array}\right.$

Figure 6 shows the variation of the squeezing force according to the displacement of the upper tool.

Three steps are observed: the first one is related to the bending of the sample, the second one to the stamping and the last one to the compression required to form the tile lug.

Assuming that the rheological model is well defined, the friction parameters could be obtained by inverse analysis of the compression force. The values of the different parameters are presented in the Table 3.

Figure 7 allows to compare the experimental compression force / displacement curve and the numerical curves obtained with the three models of friction.

The numerical model reproduces the three ranges of strain: the folding, the shaping and the compression. The difference between the numerical and experimental curves are mainly due to a different initial position of the sample between the experiment and the simulation. The use of the different friction laws, in the numerical model, shows a poor influence on the squeezing force. The squared error between the numerical curves and the experiment is lower than $6 \%$. This procedure is not suitable to perform a proper characterisation of the friction behaviour from compression tests.

\section{Damaging modelisation}

The different parameters of the rheological behaviours have been identified as well as the damage laws (with the free compression test) and the friction law (with the compression test using the technological tools).

The numerical model can be used to reproduce the technological pressing test by taking into account the damaging. The dimensions of the sample are given in Fig. 1. The volume of the sample is lower than the volume between the two tools in closed position. This allows to suppress the lateral compression.

The Fig. 8 shows the results obtained for two initial meshes. In the first case (picture on the left hand side), the initial mesh was constituted of 40,000 elements, and the final mesh by 40,100 elements. The automatic mesh refinement was not used. The element deletion algorithm leaded to a loss of volume of $22 \%$. In the second case (picture on the right hand side), the initial mesh was constituted of 8,000 elements and the final one by 75000 elements. In this simulation, the automatic mesh refinement was performed based on a damage criterion (Latham and Cockroft). The limit value was fixed to $17 \%$ of the failure threshold. The loss of volume was around $6 \%$. The CPU time was increased due to the remeshing algorithm (150 min in the first case versus in $300 \mathrm{~min}$ in the second case). However, to properly take into account the material damaging, the automatic remeshing is required to limit the loss of volume. Moreover, sharp cracks are obtained only with this method.

Figure 9 provides a qualitative comparison between the numerical shapes obtained with Forge $2009^{\circledR}$ Finite Element Methode simulation and the clay samples obtained with an interrupted pressing test.

The numerical model reproduces qualitatively the geometry of the sample according to the crosshead displacement. This allows us to validate the different parameters identified. The damaging starts at the same time in the numerical model and the experimental sample. This reveals that the failure threshold identified is correct. The damage model based on the Latham and Cockroft criterion identified previously is more relevant than the Oyane criteria. The Oyane criterion appears to be too penalizing.

\section{Conclusion and prospects}

This paper presents a study providing a numerical simulation of the shaping step of clay tiles in closed tools. The triborheological behaviour of a clay paste was studied by compression tests, highlighting an elasto-visco-plastic behaviour. The friction between the clay and a metallic surface was modelled by a Coulomb/Tresca law. The relevant damage criterion to model the cracks initiation was found to be the Latham and Cockroft criterion. The numerical simulation of a simplified geometry allowed to validate the tribo-rheological model identified. The element deletion method is relevant to model the cracks initiation but fails to simulate accurately the propagation due to a poor physical meaning. Nevertheless, an initiation criteria is sufficient to optimize the process parameters. The quality of the damage simulation was highly dependent on the elements size.

This study allowed to develop new tools and new geometries to increase the efficiency of the process. Indeed, the analysis of the numerical stress fields helped to decrease significantly the internal stresses at the end of the shaping step. Future works will include the development of a more sophisticated damage model, based on Chaboche approach. In addition, numerical 
simulation of indutrial tiles will be performed and validated using measurement made an industrial press.

Acknowledgments The present study was supported by the Terreal company. The authors would like to acknowledge this industrial partner for this support and his help.

\section{References}

1. Aoudja ZF (1988) Comportement du melange eau-argile vis à vis du procédé d'extrusion (in french), PhD Thesis, INSA de Rennes

2. Aydin I, Biglari FR, Briscoe BJ, Lawrence CJ, Adams MJ (2000) Physical and numerical modelling of a ram extrusion of paste materials: conical die entry case. Comput Mater Sci 18:141-155

3. Baran B, Ertürk T, Sarikaya Y, Alemdaroglu T (2001) Workability test method for metals applied to examine a workability measure (plastic limit) for clays. Appl Clay Sci 20:53-63

4. Bouszakis KD, Efstathiou K, Paradisiadis G, Tsouknidas A (2008) Experimental and FEM supported investigation of the wet ceramic clay extrusion for the determination of the stress distribution on the applied tools' surface. J Eur Ceram Soc 28:2117-2127

5. Cockroft MG, Latham DJ (1972) Ductility and the workability of metals pp 33-39

6. Djelal C (2001) Designing an perfecting a tribometer for the study of friction of a concentrated clay-water mixture against a metallic surface. Mater Struct 34:51-58
7. Emmerich M, Giotis A, Ozdemir M, Black T, Giannakoglou K (2002) Meta-model assisted Evolution strategies. Proceedings of the international conference on parallel problem fom nature

8. Engmann J, Servais C, Burbidge S (2000) Squeeze flow theory and applications to rheometry: a review. J Non-Newtonian Fluid Mech 328(8):601-605

9. Foudrinier E (2007) Etude numérique et experimentale du procédé d'extrusion de pâtes argileuses (in french),PhD Thesis, Ecole des Mines de Paris

10. Gagou Y, Padayodi E, Atcholi KE, Mezzane D, Fremy M-A, Saint Gregoire P (2008) Etude comparative du comportement mécanique des matrices de quatre types d'argile

11. Hoffmeister F, Back T (1990) Genetic algorithms and evolution strategic: similarities and differences. Dortmund university, Bericht, 365

12. Kocserha I, Gömze LA (2010) Friction properties of clay compounds. Appl Clay Sci 48:425-430

13. Lee HC, Choi JS, Jung KH, Im YI (2009) Application of element deletion method for numerical analyses of cracking. Process Achiev Mech Mater Eng 154-161

14. Lian G, Thornton C, Adam MJ (1993) A theoretical study of the liquid bridge forces between two rigide spherical bodies. J Colloid Interface Sci 11:138-147

15. Oyane M (1972) Criteria of ductile fracture strain pp 1507-1513

16. Ribeiro MJ, Ferreira JM, Labrincha JA (2005) Plastic behaviour of different ceramic pastes processed by extrusion. Ceram Int 31:515519

17. Soulie F (2005) Cohésion par capillarité et comportement mécanique de milieux granulaires humides, PhD Thesis 